\title{
IMPLEMENTATION OF FUZZY CONTROL OVER FES-ASSISTED LOCOMOTION FOR CVA PATIENTS
}

\author{
Ming-Han Chang ${ }^{1}$, Student Member, Yu-Leun Chen ${ }^{1}$, Student Member, \\ Kuang-Ching Wang ${ }^{1}$, Student Member, and Te-Son Kuo ${ }^{1,2}$, Senior Member \\ ${ }^{\mathrm{I}}$ Department of Electrical Engineering, National Taiwan University \\ ${ }^{2}$ The Center for Biomedical Engineering, National Taiwan University \\ E-mail: davewang@email.gcn.net.tw
}

\begin{abstract}
In this research, an Functional Electrical Stimulation (FES) system has been designed to assist Cerebral Vascular Accident (CVA) patients during the locomotion rehabilitation process. Cutaneous electrical stimulation applied over tibialis anterior muscle induced the ankle's dorsi flexion to certain degrees. An artificial neural network (ANN) was implemented to estimate the nonlinear mapping relation between stimulation levels and both ankle and knee angles for each individual subject. A rule-based fuzzy controller was used to realtime adjust the stimulating waveform so that convergence to a suitable desired flexion angle was achieved. $A$ userfriendly Windows-based interface was offered, where all fuzzy parameters and rules are adjustable, offering physical therapists' a flexible tool to find out most suitable angles for different individuals. The whole system including hardware and software have been successfully setup and tested with normal subjects in laboratory; it has also been installed to operate with a treadmill as a locomotion rehabilitation system in the hospital, where clinical tests will be held.
\end{abstract}

\section{Introduction}

Functional electrical stimulation (FES) has been long accepted to improve muscular functional capabilities for patients of different categories. So far, most related researches with FES applications have been focused on Spinal Cord Injury (SCI) patients' rehabilitation[1, 2]. However, patients suffering Cerebral Vascular Accident (CVA) also make up a large disabled population, while less attention has been paid to them[3]. One of the reasons CVA patients were less frequently chosen as subjects was due to some difficulties in control considerations. First of all, CVA patients often still possess partial sensory inputs and voluntary movements. Considering patients' sensus, stimulation current has to be kept low to avoid pain experience, while the frequently adopted cutaneous stimulation requires higher current levels. Although stimulation current could be kept low and more precise motion control could be achieved by using implant electrode techniques, it is apparently improper for CVA patients, considering their remaining sensory inputs.

This research aimed to offer a suitable FES system to assist CVA, especially hemiplegic patients during locomotion rehabilitation process. During patients' locomotion training, electrical stimulation was applied to assist both standing phase and swinging phase processes. The system was designed to accomplish both tasks, and swinging phase has been tested in the laboratory. Stimulation was applied cutaneously over the tibialis anterior muscle to induce the ankle's dorsi flexion to smooth the patient's swinging phase process. To achieve the desired flexion angle correctly, realtime angle monitoring and automatic stimulation adjustments were implemented.

Artificial neural networks (ANN) and fuzzy inference systems have been frequently used to serve control purposes in changing environments of uncertainty and imprecision[4]. This FES assisting system adopted these two techniques to achieve flexion angle control. An ANN was used to establish the nonlinear mapping relation between stimulation current levels and resulted flexion angles for each individual. A rulebased fuzzy controller was used to infer stimulation adjustment decisions. Through the Windows-based interface, the desired angle could be set to suitable values depending on physical therapists' decision, and the fuzzy rules could also be defined as the original PID-like rules[5] or adjusted easily by therapists considering patients muscular abilities' recovery condition with time. Therefore, our main purpose of finding suitable angle and stimulation levels for each individual was achieved for either phase of locomotion. The system's configuration is shown in fig.1. Body weight support served to resolve CVA patients' balance problem.

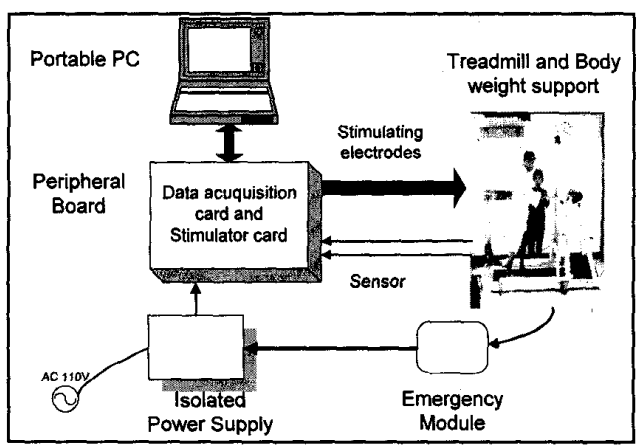

Fig.1. System Configuration 


\section{Methods}

The system consisted of a host PC running the control program, a stimulating portion, and a sensor feedback portion as shown in fig.2. The control program computed all channels' stimulation levels and passed them to the stimulating portion, which actually output the stimulating current. The stimulated result, which was evaluated as angles, were fed back through position sensors providing information for further adjustment.

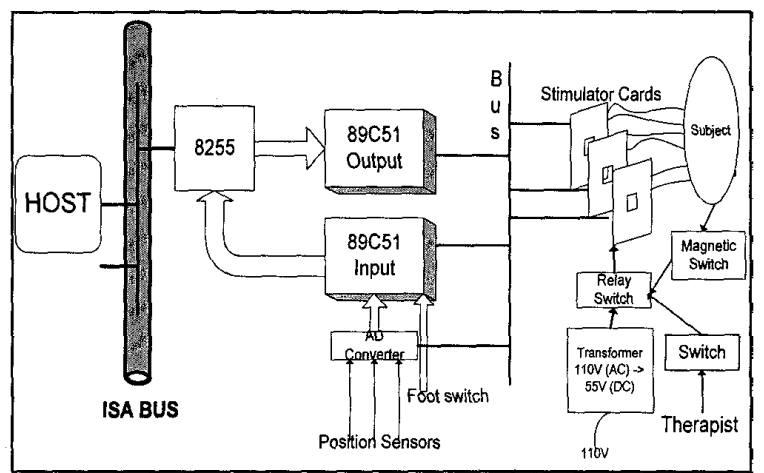

Fig.2. System Block Diagram

\section{a. Host software algorithm}

Fuzzy inference serves the core task of adjusting stimulating waveform. The system adopted the Mamdanistyle fuzzy inference method, following the Min-Min-Max process $[6,7]$. Besides, a linear function was used to fuzzify the input, and the centroid method was used to defuzzify the inference result. The software procedure could be briefly stated as three steps (fig. 3 ).

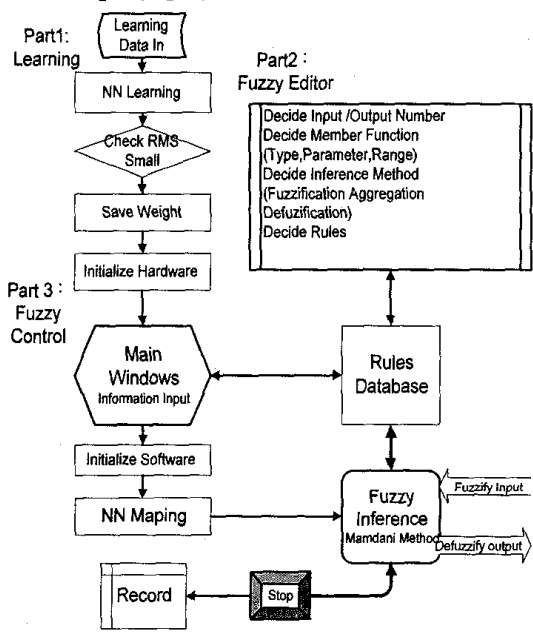

Fig.3. Host Software Flow Chart

First, the patient's mapping relations between both ankle and knee angles and stimulation levels were recorded through simple testing. These relations would be mapped by an ANN.
Secondly, the fuzzy rules, input and output numbers, member functions, and specific strategies were defined with therapists' opinions (fig.4). The system started online operation on the third step. All necessary fuzzy information, desired angles, and stimulation waveforms were chosen through the main window and the stimulation and real-time adjustment loop began (fig.5). Summarily, the ANN offered a jump-start initial value to speed up the convergence, and the fuzzy controller offered a smooth control over the stimulation levels.

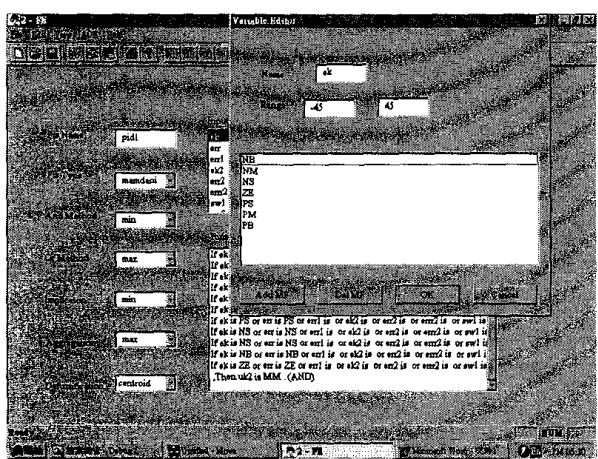

Fig.4. Fuzzy Editor Window

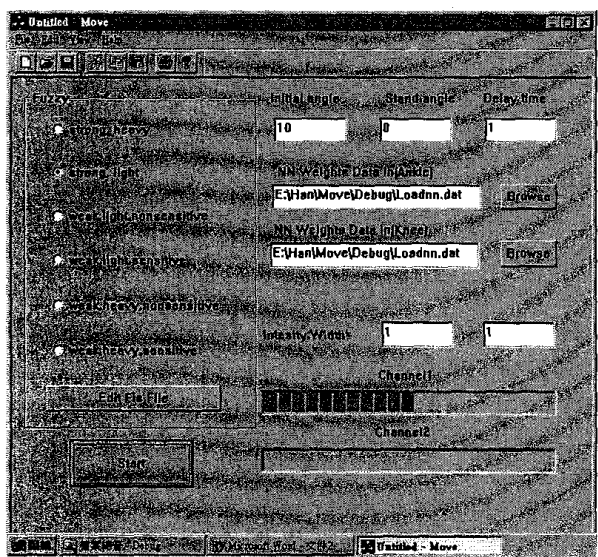

Fig.5. Main Window

b. Hardware description

As mentioned, the whole system consisted of three portions. The main algorithms were run inside the host PC, while two single chip controllers handle the stimulation output and sensor data acquisition respectively. Three magnetic position sensors achieved the angle monitoring process. The switching between two phases of locomotion was detected by three foot switches and dealt with inside the software program.

\section{c. Safety considerations}

Electrical isolation is the most important consideration. Three stages of isolation were implemented. The power supply module was isolated magnetically through transformers. The power flow was further isolated through 
optical couplers before driving the system circuits. Finally, the stimulating channels were implemented by relays, offering a definitely isolated constant current source. Furthermore, the whole system could be manually stopped through either software or hardware designs.

\section{Results}

For now, the system has been tested under both DOS environment and Windows95 environment. Under DOS environment, conventional PID control was adopted to test the ankle angle convergence rate. Totally 36 trials were tested among 3 healthy subjects. Under 22 stimulating cycles/sec, the average convergence rate was found to be 48 cycles. Under Windows95 environment, the ANN mapping was first tested to estimate initial values for the stimulation level. Randomly choosing desired ankle angles from 0 to 15 degrees, constant current levels were computed, and resulted angles were estimated with an error range of +2 to -3 degrees. Finally, the fuzzy controller was combined with the ANN, totally 30 trials were tested among 2 healthy subjects in the laboratory. Using simple fuzzy rules, 21 trials were converged in $0.61 \mathrm{sec}$. The main purpose of the system was to assist the locomotion rehabilitation in the hospital; therefore, it should be evaluated according to the improvement of the patient's locomotion ability. More clinical tests will be held in the hospital, while the convergence rate estimated so far was considered appropriate.

\section{Discussion}

Test results compared the conventional PID control and the ANN-combined fuzzy control performance. The former performed a lower convergence rate, since a mathematical model for the nonlinear property of a physiological system was not available. The ANN-combined fuzzy system could achieve a faster convergence, which was appropriate for the locomotion process. Besides the control performance, the system was also expected to provide physical therapists a friendly interface, therefore better strategies could be designed for different CVA patients to achieve formal locomotion finally. So far all tests were conducted with healthy subjects, who possessed even more sensitive sensory input and voluntary movements, and the stimulated motions were observed smooth, convergent, and under appropriate stimulation level. Therefore, when CVA patients' tests shall be held, the control strategies could be expected applicable, since their sensory inputs will be less sensitive than normal patients, less voluntary motion will occur, and a wider range of stimulation levels will be available.

\section{Acknowledgement}

The research was funded by the National Science Council, China (Taiwan), under Grant NSC 87-2213-E-002-067, 1998.

\section{Reference}

1. H. J. Chizeck, R. Kobetic, E. B. Marsolais, J. J. Abbas,
"Control of functional neuromuscular stimulation system for standing and locomotion in paraplegics, " Proc. IEEE, Vol. 76, no. 9, pp 1155-1165, 1988.

2. M. Moynahan, C. Mullin, J. Cohn, C. A. Burns, E. E. Halden, R. J. Triolo, R. R. Betz, "Home use of a functional electrical stimulation system for standing and mobility in adolescents with spinal cord injury, "Arch. Phys. Med. Rehabil., Vol 77, pp 1005-1013, 1996.

3. F. M. Gollen, D. T. Wade, C. M. Bradshaw, "mobility after stroke: reliability of measures of impairment and disability," Int. Disability Study, Vol. 12, pp. 6-9, 1990.

4. J.-S. R. Jang, C.-T. Sun, E. Mizutani, "Neuro-fuzzy and soft computing," Prentice-Hall Inc., 1997.

5. J. J. Chen, Y. N. Yu, D. G. Hung, B. T. Ann, G. C. Chang, "Applying fuzzy logic to control of cycling movement induced by functional electrical stimulation," IEEE Trans. Rehab. Eng., Vol. 5, no. 2, pp. 158-169, 1997.

6. E. H. Mamdani, S. Assilian. "An experiment in linguistic synthesis with a fuzzy logic controller," Inter. J. ManMach. Studies, Vol. 7, no.1, pp. 1-13, 1975.

7. E. H. Mamdani, B. R. Gaines, "Fuzzy reasoning and its applications," Academic Press, 1981. 